\title{
Mediastinoscopic esophagectomy with lymph node dissection using a bilateral transcervical and transhiatal pneumomediastinal approach
}

\author{
Yutaka Tokairin ${ }^{1,2}$, Yasuaki Nakajima², Kenro Kawad ${ }^{2}$, Akihiro Hoshin $^{2}$, Takuya Okada ${ }^{2}$, Toshihiro \\ Matsui $^{2}$, Kazuya Yamaguchi ${ }^{2}$, Kagami Nagai², Yusuke Kinugasa² \\ 'Department of Surgery, Toshima Hospital Tokyo Metropolitan Health and Hospitals Corporation, Tokyo 173-0015, Japan. \\ 2Department of Gastrointestinal Surgery, Tokyo Medical and Dental University, Tokyo 113-8510, Japan.
}

Correspondence to: Dr. Yutaka Tokairin, Department of Surgery, Toshima Hospital Tokyo Metropolitan Health and Hospitals Corporation, 33-1 Sakaecho, Itabashi-ku, Tokyo 173-0015, Japan. E-mail: tokairin.srg1@tmd.ac.jp

How to cite this article: Tokairin Y, Nakajima Y, Kawad K, Hoshin A, Okada T, Matsui T, Yamaguchi K, Nagai K, Kinugasa Y. Mediastinoscopic esophagectomy with lymph node dissection using a bilateral transcervical and transhiatal pneumomediastinal approach. Mini-invasive Surg 2020;4:32. http://dx.doi.org/10.20517/2574-1225.2020.23

Received: 17 Feb 2020 First Decision: 17 Mar 2020 Revised: 24 Mar 2020 Accepted: 1 Apr 2020 Published: 16 May 2020

Science Editor: Itasu Ninomiya Copy Editor: Jing-Wen Zhang Production Editor: Tian Zhang

\begin{abstract}
We developed a method for mediastinoscopic esophagectomy via a bilateral transcervical and transhiatal approach under pneumomediastinum as a less-invasive radical operation. The right recurrent nerve is first identified using an open approach, and the right cervical paraesophageal lymph nodes and part of the right recurrent nerve lymph nodes are dissected, after which pneumomediastinum is initiated. The upper thoracic paraesophageal lymph nodes and right recurrent nerve lymph nodes are dissected along the right vagus nerve. The dorsal side of the esophagus is dissected along the visceral sheath taking care to avoid thoracic duct injury and is then dissected along the vascular sheath in front of the descending aorta. The esophagus is dissected from the trachea at the caudal side of the aortic arch, and then dissected along the ventral side of the left main bronchus, reaching the pulmonary artery. Finally, the right recurrent nerve lymph nodes around the right subclavian artery are completely retrieved. The left cervical approach is almost the same as that via the right side. The dorsal side of the esophagus is almost dissected along the visceral sheath with a right transcervical approach. The subaortic arch to the left tracheobronchial lymph nodes are dissected using the crossover technique. These lymph nodes are easily dissected by cutting the left and ventral side of the lymph nodes because the caudal side is already dissected in the right transcervical approach. A bilateral (especially right trans-cervico-pneumomediastinal) approach is useful for bilateral upper mediastinal lymph node dissection and esophagectomy.
\end{abstract}

Keywords: Minimally invasive esophagectomy, cervical approach, esophageal cancer, mediastinoscopic esophagectomy, pneumomediastinum, mediastinoscopic esophagectomy with lymph node dissection

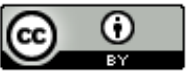

(C) The Author(s) 2020. Open Access This article is licensed under a Creative Commons Attribution 4.0 International License (https://creativecommons.org/licenses/by/4.0/), which permits unrestricted use, sharing, adaptation, distribution and reproduction in any medium or format, for any purpose, even commercially, as long as you give appropriate credit to the original author(s) and the source, provide a link to the Creative Commons license, and indicate if changes were made.

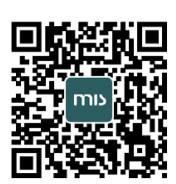




\section{INTRODUCTION}

Conventional radical esophagectomy through right thoracotomy is one of the most invasive procedures. It is important to reduce the invasiveness of this procedure.

Conventional radical esophagectomy was previously performed for the treatment of mucosal esophageal cancers for patients diagnosed with not only advanced esophageal cancer but also T1a No Mo cStage I according to the Union for International Cancer Control TNM Classification (eighth edition) ${ }^{[1]}$; however, endoscopic submucosal dissection has come to be performed for patients with T1a No Mo cStage I as minimally invasive treatment and the methods have been well established ${ }^{[2]}$.

On the other hand, the esophagectomy with dissection of the mediastinal and abdominal lymph nodes is needed for the treatment of thoracic esophageal cancer with invasion of the submucosal layer or deeper layers. For the abovementioned reason, hybrid surgery consisting of a two-field abdominal-thoracic operation (called the Ivor-Lewis procedure) was developed ${ }^{[3]}$. In Western countries, abdominal esophageal cancer and esophagogastric junctional cancers are well observed and later histological types are frequently diagnosed as "adenocarcinoma". Thus, this procedure is considered reasonable because esophageal cancers at these locations are rarely associated with upper mediastinal and cervical lymph node metastasis. Esophageal cancers in the thoracic esophagus are frequently associated with upper mediastinal and cervical lymph node metastasis. Thus, this procedure is not suitable for these esophageal cancers. To resolve this problem, McKeown developed total esophagectomy with three-field lymph node dissection ${ }^{[4]}$.

Currently, radical esophagectomy through right thoracotomy has changed to esophagectomy via a thoracoscopic or laparoscopic approach, including robot assisted surgery, which reduces the invasiveness of the procedure by decreasing the destruction of thoracic and abdominal walls ${ }^{[5-11]}$. However, this method mandates the use of one-lung ventilation, some destruction of the thoracic wall, or prone positioning.

Conventional transhiatal esophagectomy has been performed and mediastinoscopic esophagectomy has been developed. These procedures are also recognized as types of minimally invasive esophagectomy (MIE) ${ }^{[12-14]}$. However, due to the blind maneuvering in the upper and middle mediastinum that is necessary in this procedure and the difficulty of systematic lymph node dissection, it is usually only applied in limited cases, such as cases of esophagogastric junction cancer, very early-stage cancers, or some cases of advanced thoracic esophageal cancer for the purpose of palliative resection ${ }^{[12]}$.

Recently, we developed and reported the performance of "mediastinoscopic esophagectomy with lymph node dissection (MELD)" under pneumomediastinum using a bilateral transcervical and transhiatal approach, as a method of radical esophagectomy ${ }^{[15-18]}$. This procedure achieves curative radical esophagectomy with minimal invasiveness. Upper mediastinal lymph node dissection has been performed using bilateral open cervical surgery and a left transcervical and transhiatal pneumomediastinal approach in some institutions ${ }^{[19,20]}$. However, in the results of our clinical trial, the right recurrent nerve lymph nodes, the upper thoracic paraesophageal lymph nodes, and the subaortic arch to the left tracheobronchial lymph nodes could not be completely dissected using the left transcervical approach alone ${ }^{[17,18]}$. We are therefore of the opinion that a right cervical pneumomediastinal approach is necessary to achieve the complete dissection of these lymph nodes. We herein describe the surgical technique using a bilateral (especially right cervico-pneumomediastinal) approach.

\section{TECHNIQUE}

In the MELD procedure, the right cervical approach is performed first, followed by the left cervical approach and laparoscopic-transhiatal approach. 


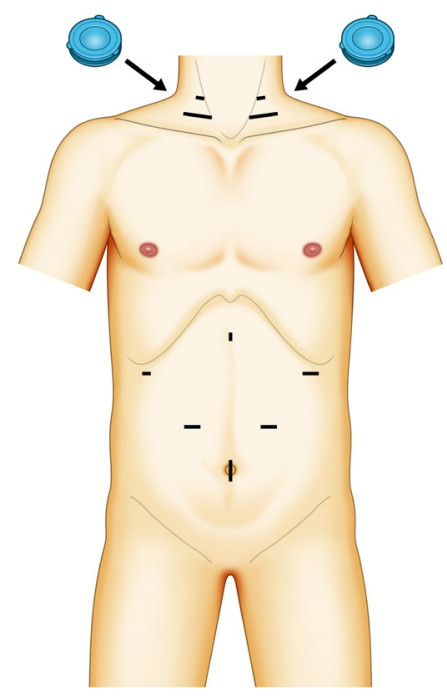

Figure 1. The location of the ports and single-port laparoscopic access devices. This schematic illustration shows single-port laparoscopic access devices in the bilateral cervical area and ports in the abdominal area. A single-port laparoscopic access device with three 5-mm trocars is placed in a triangle configuration. Approximately $3 \mathrm{~cm}$ above this device, a $5-\mathrm{mm}$ trocar is inserted as a mediastinoscopic port

\section{Right cervical approach}

A right cervical collar incision (approximately $6 \mathrm{~cm}$ ) is made $3 \mathrm{~cm}$ above the right clavicle, and sufficient working space is created between the tracheoesophageal groove and right carotid sheath. The right recurrent nerve is first identified using an open approach and part of the right cervical paraesophageal lymph nodes and the right recurrent nerve lymph nodes between the tracheal wall and the right recurrent nerve are dissected.

After a single-port laparoscopic access device (Lap Protector; Hakko Corporation, Nagano, Japan) is inserted in the wound to exclude the right lobe of the thyroid gland, the cervical wound is sutured over a length of approximately $4 \mathrm{~cm}$. The device is placed at the dorsal side of the sternohyoid and the scapulohyoid muscles at the cranial side, at the inner border of the sternal head of the sternocleidomastoid muscle on the outside, and at the dorsal side of the clavicular head of the sternocleidomastoid muscle on the caudal side. Next, an EZ access (Hakko Corporation, Nagano, Japan), through which three 5-mm trocars are placed in a triangle configuration [Figure 1], is attached. Then, pneumomediastinum (to $8 \mathrm{mmHg}$ ) is established with $\mathrm{CO}_{2}$. A $5-\mathrm{mm}$ trocar is inserted as a mediastinoscopic port approximately $3 \mathrm{~cm}$ above this device; we can observe the whole forceps movement from the beginning of forceps insertion. A laparoscopic forceps and a LigaSure ${ }^{\mathrm{TM}}$ Maryland (Medtronic, Minneapolis, MN) are inserted between the tracheal wall and the internal side of the right recurrent nerve [Figure $2 \mathrm{~A}$ and $\mathrm{B}$ ]. The upper thoracic paraesophageal lymph nodes and the right recurrent nerve lymph nodes are dissected along the right vagus nerve with attention paid to avoid injury of the right mediastinal pleura [Figure 3]; then, the proximal portion of the azygos vein and the right bronchial artery are dissected [Figure 4A and B]. Next, the dorsal side of the esophagus is dissected along the visceral sheath with attention paid to avoid the injury of the thoracic duct, which is located on the dorsal side of the visceral sheath [Figure 5]. The esophagus is dissected along the vascular sheath in front of the descending aorta, because the visceral sheath becomes unclear on the caudal side of the bifurcation of the trachea [Figure $4 \mathrm{~A}$ and B]. During dissection, the proper esophageal artery is double sealed and cut using a LigaSure ${ }^{\mathrm{TM}}$ Maryland. Then, the esophagus is dissected from the trachea on the caudal side of the aortic arch. In some cases, the bronchooesophagus muscle is developed and attention should be paid to avoid injury of the membranous portion of the trachea. Next, dissection is performed along the ventral side of the left main bronchus, reaching to the pulmonary artery, in order to determine the caudal side of the lymph nodes in the subaortic arch to the 

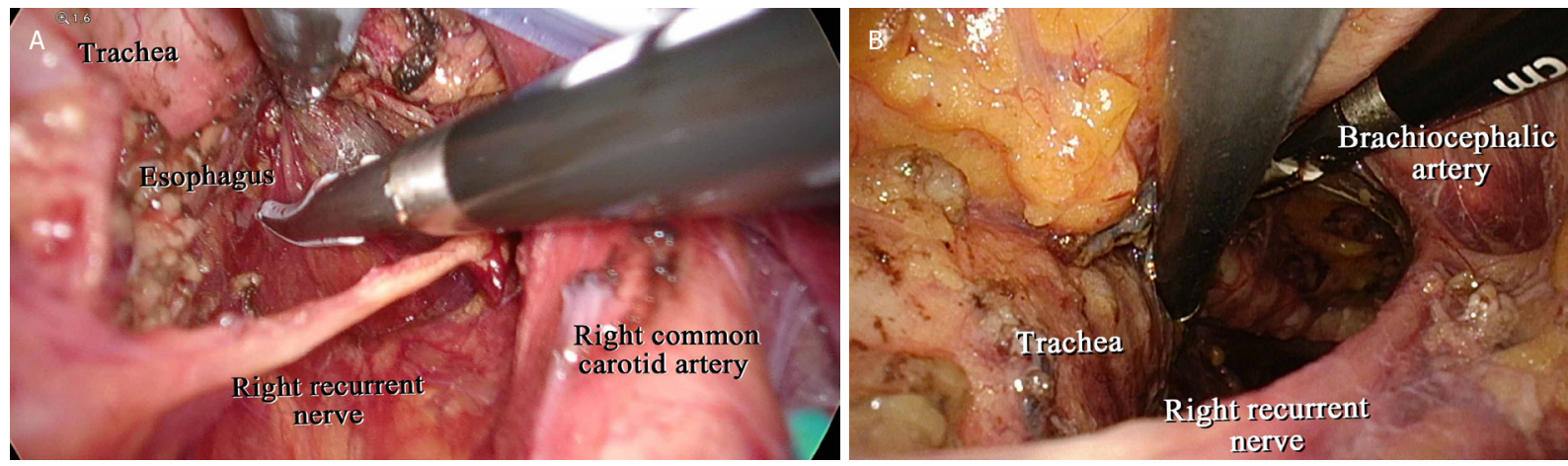

Figure 2. $A, B$ : dissection via a right transcervical approach under pneumomediastinum. The laparoscopic forceps and a vessel sealing system are inserted between the tracheal wall and the internal side of the right recurrent nerve

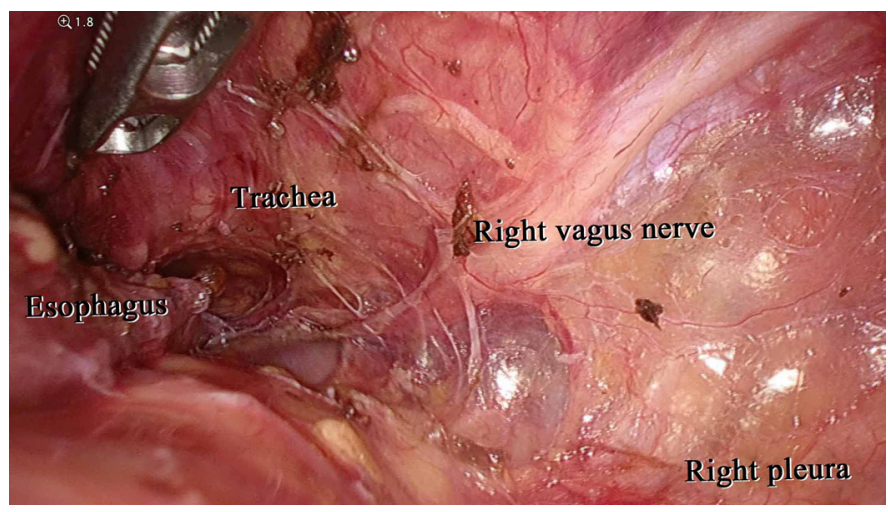

Figure 3. Dissection of the right side of the esophagus via a right transcervical approach under pneumomediastinum. The upper thoracic paraesophageal lymph nodes and right recurrent nerve lymph nodes are dissected along the right vagus nerve with attention paid to avoid injury to the right mediastinal pleura
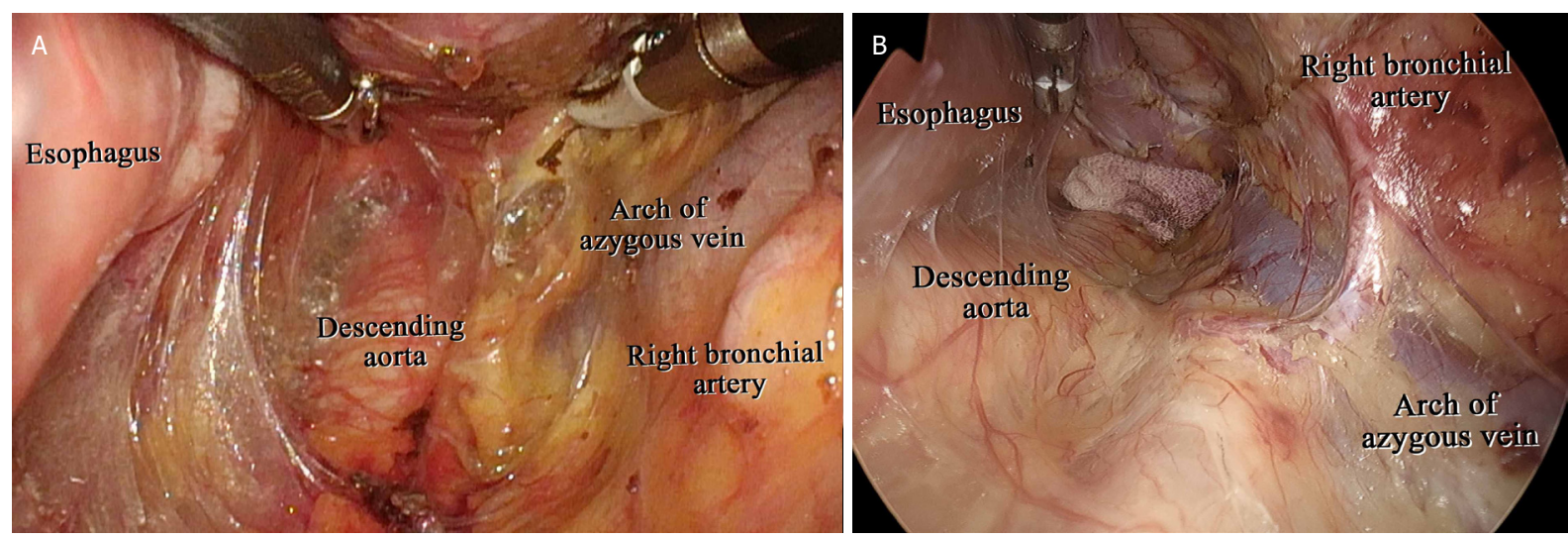

Figure 4. $A$, B: dissection of the right dorsal side of the esophagus via a right transcervical approach under pneumomediastinum. The proximal portion of the azygos vein and the right bronchial artery is observed along the right mediastinal pleura. The esophagus is dissected along the vascular sheath in front of the descending aorta, because the visceral sheath becomes unclear on the caudal side of the bifurcation of the trachea

left tracheobronchial region, which should be dissected. To make it easy to dissect the left recurrent nerve lymph nodes, the left recurrent nerve is exposed in the right transcervical pneumomediastinal approach [Figure 6]. 


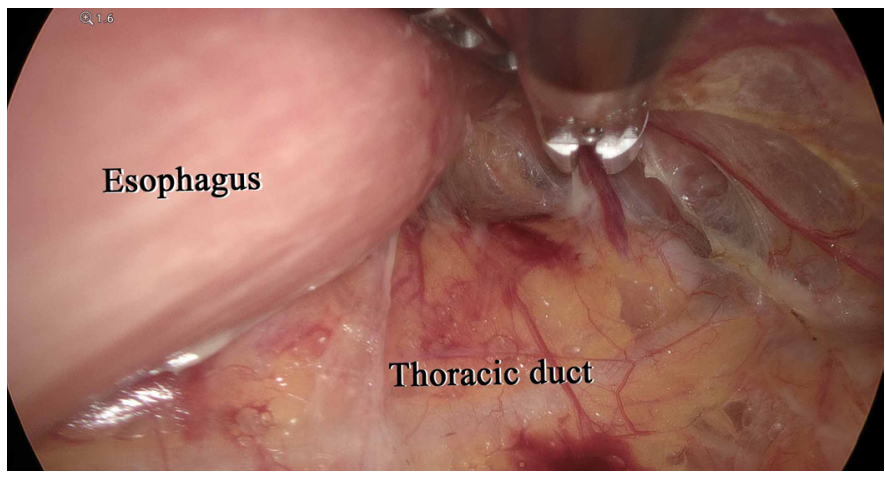

Figure 5. Dissection of the dorsal side of the esophagus via a right transcervical approach under pneumomediastinum. The dorsal side of the esophagus is dissected along the visceral sheath with attention paid to avoid injury to the thoracic duct, which is located on the dorsal side of the visceral sheath

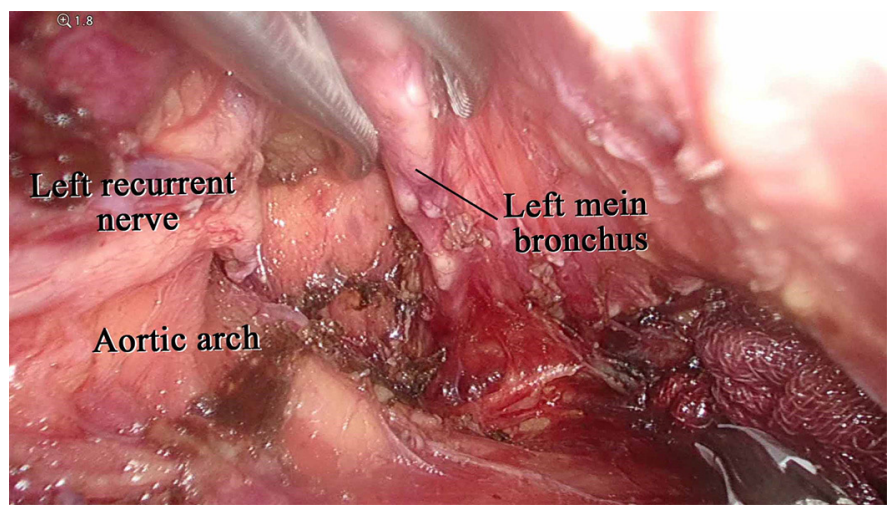

Figure 6. Dissection of the cartilage of the left main bronchus via a right transcervical approach under pneumomediastinum. Dissection of the subaortic arch to the left tracheobronchial lymph nodes is performed along the ventral side of the left main bronchus reaching to the pulmonary artery to determine the caudal side of the lymph nodes in the subaortic arch to the left tracheobronchial region, which should be dissected. To enable the easy dissection of the left recurrent nerve lymph nodes, the left recurrent nerve is exposed via the right transcervical pneumomediastinal approach

Finally, after the common carotid artery is pulled outward and the esophagus is pulled to the left side, the right recurrent nerve lymph nodes around the right subclavian artery are completely retrieved [Figure 7]. Under pneumomediastinum, these lymph nodes can be completely dissected.

\section{Left cervical approach}

A left cervical collar incision and scope port site are made symmetrically. After identifying the cranial portion of the left recurrent nerve, which is located on the dorsal side of the inferior part in the left lobe of thyroid gland, some of the left cervical paraesophageal lymph nodes and left recurrent nerve lymph nodes are dissected by the open method.

After a single-port laparoscopic access device is inserted into the wound, the cervical wound is sutured over a length of approximately $4 \mathrm{~cm}$.

The device is placed at the dorsal side of the sternohyoid and the scapulohyoid muscles on the cranial side, at the inner border of the sternal head of the sternocleidomastoid muscle on the outside and at the dorsal side of the clavicular head of the sternocleidomastoid muscle on the caudal side. After attaching an EZ access and inserting a $5-\mathrm{mm}$ trocar as a mediastinoscopic port, left transcervical pneumomediastinum is applied similarly to the right side. First, the dissected layer on the dorsal side of the esophagus and from the 


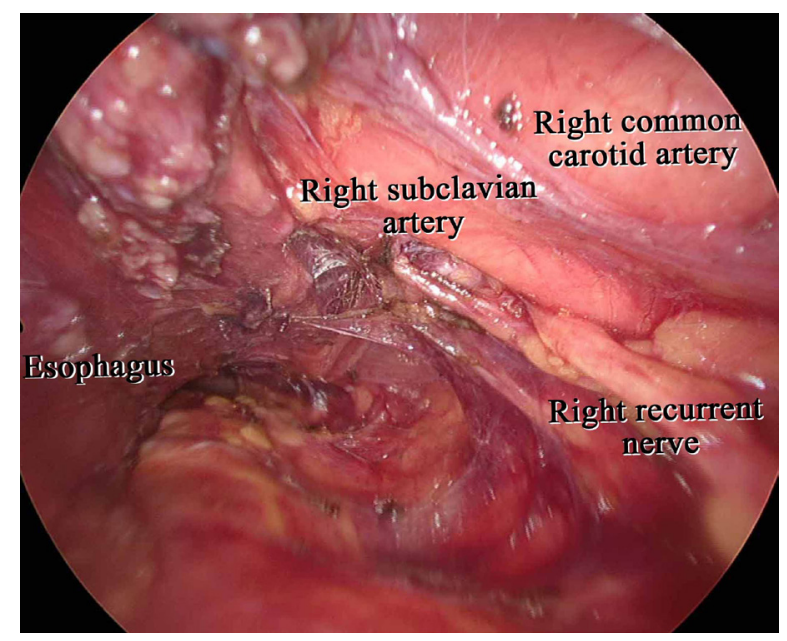

Figure 7. Dissection of the right recurrent nerve lymph nodes via a right transcervical approach under pneumomediastinum. After the right common carotid artery is pulled outward and the esophagus is pulled to the left, the right recurrent nerve lymph nodes around the right subclavian artery are completely retrieved
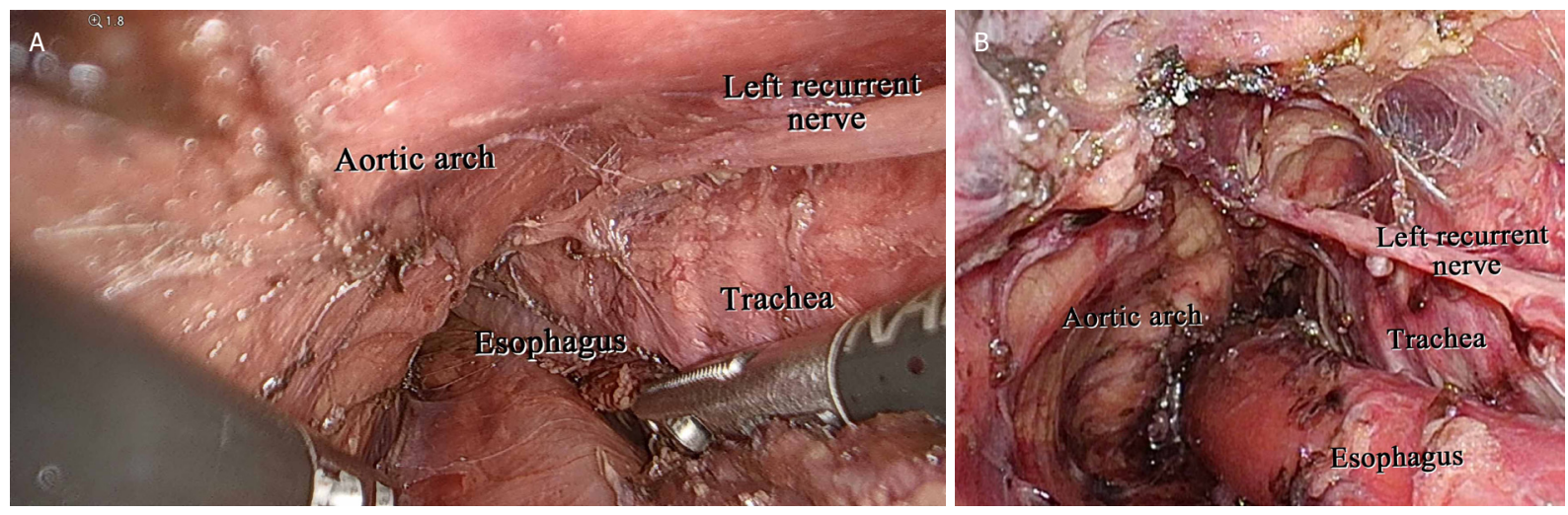

Figure 8. Dissection of the left recurrent nerve lymph nodes via a left transcervical approach under pneumomediastinum. A: the left recurrent nerve lymph nodes are dissected along the dorsal side of the left recurrent nerve; $B$ : the ventral side of the left recurrent nerve lymph nodes are dissected along the left recurrent nerve and the tracheal branch of the left recurrent nerve is preserved as much as possible

right side is checked and dissection is performed to near the aortic arch with attention paid to avoid injury to the visceral layer. In many cases, the esophagus has already been mostly dissected by the right transcervical approach. Next, the esophagus is dissected from the left subclavian artery, reaching to the aortic arch.

The ventral side of the esophagus is dissected from the left side of the membranous portion of the trachea, the left main bronchus, and the aortic arch. The left recurrent nerve lymph nodes are dissected along the dorsal side of the left recurrent nerve [Figure $8 \mathrm{~A}$ ]. Because of this procedure, the left recurrent nerve and the ventral side of the left recurrent nerve lymph nodes are attached to the left side of the trachea. Because the left recurrent nerve at the recurrent portion on the aortic arch has already been exposed via the right transcervical approach, the recurrent nerve is easily detected. We think that this method is useful for preventing left recurrent nerve injury.

Next, the ventral side of the left recurrent nerve lymph nodes is dissected along the left recurrent nerve [Figure $8 \mathrm{~B}$ ], and the tracheal branch of the left recurrent nerve is preserved as much as possible ${ }^{[21]}$. We consider that this method is useful for two reasons: First, appropriate tension can be placed on the left 


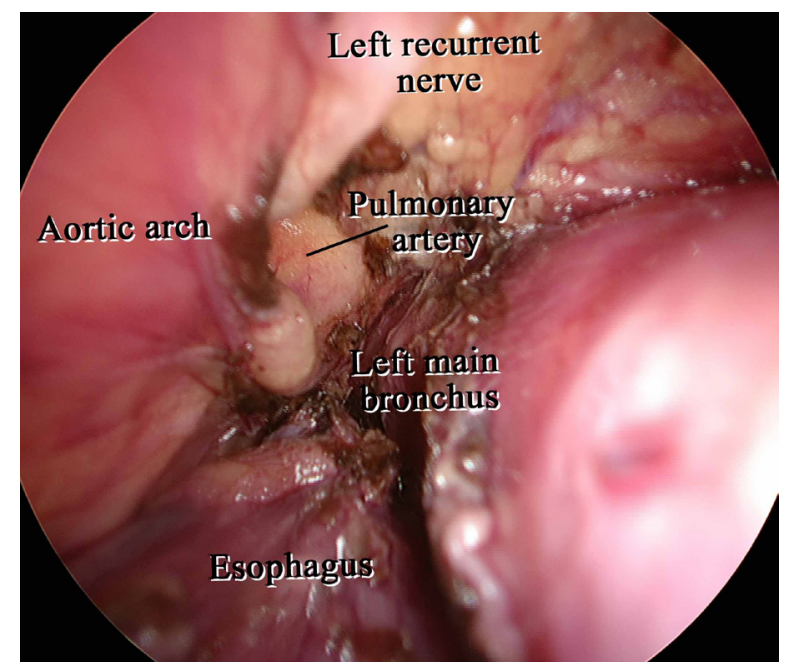

Figure 9. Dissection of the subaortic arch to the left tracheobronchial lymph nodes via a left transcervical approach under pneumomediastinum. These lymph nodes can easily be dissected by cutting only the left and ventral side of these lymph nodes while pulling the esophagus toward the right side because the caudal and dorsal side of these lymph nodes have been already dissected via a right transcervical approach

recurrent nerve lymph nodes and the dissection of these lymph nodes can be easily performed because the left recurrent nerve is attached to the tracheal wall. Second, the branch of the left recurrent nerve is considered useful for preserving the cough reflex.

\section{Dissection of the subaortic arch to the left tracheobronchial lymph nodes}

The subaortic arch to the left tracheobronchial lymph nodes is dissected using the crossover technique, as described previously ${ }^{[15-18]}$. The crossover technique consists of the transhiatal, right transcervical, and/or left transcervical approach and is suitable for dissection in narrow and relatively deep operative fields, as the approach can be made from two directions. We use this technique when dissecting the subaortic arch to the left tracheobronchial lymph nodes. First, the caudal and dorsal sides of these lymph nodes are dissected via the right transcervical approach. The lymph nodes can then dissected by cutting only their left and ventral sides with the esophagus pulled toward the right via the left transcervical approach [Figure 9].

\section{Laparoscopic and transhiatal approach}

The ports are located in an inverted trapezoidal shape, as described in Figure 1, in accordance with laparoscopic gastrectomy. Pneumoperitoneum with $\mathrm{CO}_{2}$ (to $10 \mathrm{mmHg}$ ) is then introduced. After the dissection of the upper abdominal lymph nodes and gastric conduit mobilization, the esophagus is cut at cranial side as much as possible. The suture thread at the cut end of the esophagus is pulled to provide traction for the dissection of the middle to lower mediastinal lymph nodes, including the subcarinal lymph nodes under pneumomediastinum [Figure 10]. In our institute, this is performed using totally endoscopic surgery. We often use the ENSEAL ${ }^{\bullet}$ G2 Articulating Tissue Sealer (Ethicon Endo-surgery, Cincinnati, Ohio), which is a curved long bipolar sealer, because the subcarinal nodes are difficult to dissect from abdominal ports.

\section{Range of the operation field in the cervical and transhiatal approach under pneumomediastinum}

The dorsal side of the esophagus from the cervical area to near the pulmonary vein is easily dissected via the cervical pneumomediastinal approach. On the other hand, the subcarinal lymph nodes are easily dissected via a transhiatal pneumomediastinal approach rather than a cervical approach. This is for the following reasons. First, these lymph nodes can be easily dissected en bloc with the esophagus via transhiatal approach but not via a cervical approach. Second, bleeding from the subcarinal lymph nodes connected to the pretracheal lymph nodes cannot be stopped easily via a cervical approach. 


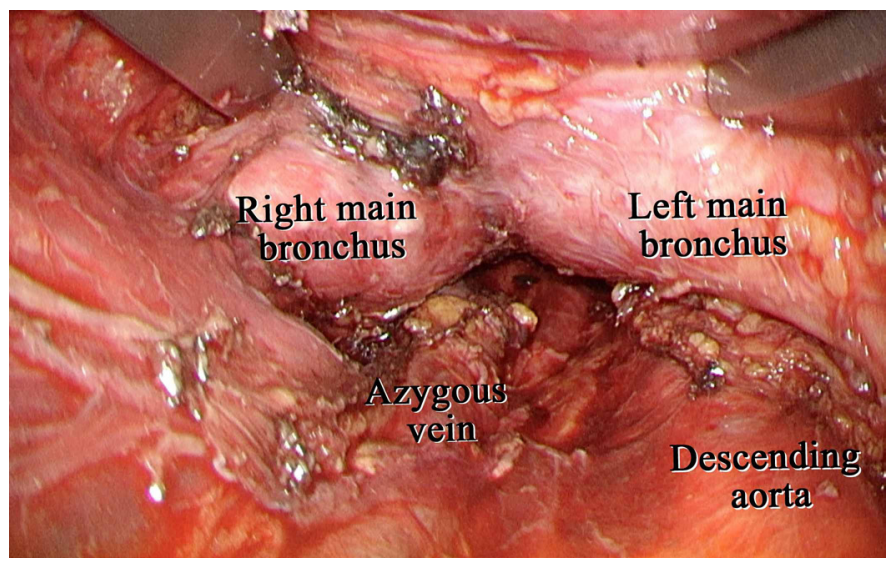

Figure 10. Subcarinal lymph node dissection via a transhiatal approach under pneumomediastinum. The middle to lower mediastinal lymph nodes, including the subcarinal lymph nodes, are dissected under pneumomediastinum via a transhiatal approach

We consider the "crossover technique" to be useful for the dissection of the lymph nodes near the bilateral main bronchus because these lymph nodes are located in the deepest area via both the cervical and transhiatal approaches.

After the pneumomediastinal procedure, the bilateral remnant cervical paraesophageal lymph nodes and supraclavicular lymph nodes are dissected using an open method.

The median total number of dissected lymph nodes in the cervico-mediastinal region identified with a mediastinoscope was 36 (range 22-76) in 10 cases treated using the MELD procedure in our institute.

\section{Postoperative outcomes}

This operation using the bilateral cervical approach under pneumomediastinum was performed for 10 cases. The median mediastinoscopic operation time was 312 (299-336) mins and the median blood loss was 476 (203-667) $\mathrm{mL}$. The median postoperative stay was 15.5 (14.0-16.8) days.

\section{DISCUSSION}

The MELD procedure is considered to have several advantages over other approaches. First, this procedure does not require one-lung ventilation or a prone position. Second, this procedure requires only bi-cervical and abdominal ports, thus no thoracic wounds are made, and the surgical wounds are very small. Finally, the view via the right transcervical approach under pneumomediastinum is similar to that via the right transthoracic approach.

Concerning the surgical outcomes, the blood loss was slight, and the postoperative stay was short. In addition, dissection of the mediastinal lymph nodes using our MELD procedure was not inferior to that with thoracoscopic esophagectomy ${ }^{[22]}$. These results and the known benefits thus indicate that this procedure is promising and expected to become prevalent in the near future.

However, this procedure is considered to have some disadvantages as well. This procedure requires a long operation time, and recurrent nerve palsy is more frequently observed than with thoracoscopic esophagectomy ${ }^{[10]}$. In our institute, recurrent nerve palsy was recognized in about $40 \%$ of cases treated with this procedure. However, most cases recovered within six months. We speculate that recurrent nerve palsy occurred for reasons such as extension of the recurrent nerve and crush injury of the recurrent nerve. Evaluating the recurrent nerves and improving the surgical procedure using NIM nerve monitoring systems is expected to help prevent recurrent nerve palsy ${ }^{[23]}$. 
We consider dissection of the mediastinal lymph nodes using our MELD procedure to be sufficient. However, whether the mediastinal lymph nodes are truly sufficiently dissected using this approach should be confirmed in the next stage.

\section{CONCLUSION}

We conclude that a bilateral cervico-pneumomediastinal approach is useful for performing bilateral upper mediastinal lymph node dissection and esophagectomy.

\section{DECLARATIONS}

\section{Authors' contributions}

Conception and design of the study: Tokairin Y, Nagai K

Informed consent of the patients was obtained by Tokairin Y, Nakajima Y, Kawad K, Hoshin A, Okada T, Matsui T, Yamaguchi K

Drafting of manuscript: Tokairin Y

Revision and final manuscript: Tokairin Y, Nagai K

General supervision: Kinugasa Y

\section{Availability of data and materials}

Not applicable.

\section{Financial support and sponsorship}

None.

\section{Conflicts of interest}

All authors declared that there are no conflicts of interest.

\section{Ethical approval and consent to participate}

Not applicable.

\section{Consent for publication}

Written informed consent for publication was obtained as appropriate.

\section{Copyright}

(c) The Author(s) 2020.

\section{REFERENCES}

1. Brierley JD, Gospodarowicz MK, Wittekind C. eds. TNM Classification of Malignant Tumours, 8th Edition.Oxford: Wiley-Blackwell; 2017. pp. 57-62.

2. Tachimori Y, Ozawa S, Numasaki H, Ishihara R, Matsubara H, et al.; Registration Committee for Esophageal Cancer of the Japan Esophageal S. Comprehensive registry of esophageal cancer in Japan, 2012. Esophagus 2019;16:221-45.

3. Lewis I. The surgical treatment of carcinoma of the oesophagus; with special reference to a new operation for growths of the middle third. Br J Surg 1946;34:18-31.

4. McKeown KC. Trends in oesophageal resection for carcinoma with special reference to total oesophagectomy. Ann R Coll Surg Engl 1972;51:213-39.

5. Biere SS, van Berge Henegouwen MI, Maas KW, Bonavina L, Rosman C, et al. Minimally invasive versus open oesophagectomy for patients with oesophageal cancer: a multicentre, open-label, randomised controlled trial. Lancet 2012;379:1887-92.

6. Akaishi T, Kaneda I, Higuchi N, Kuriya Y, Kuramoto J, et al. Thoracoscopic en bloc total esophagectomy with radical mediastinal lymphadenectomy. J Thorac Cardiovasc Surg 1996;112:1533-40.

7. Nguyen NT, Follette DM, Lemoine PH, Roberts PF, Goodnight JE Jr. Minimally invasive Ivor Lewis esophagectomy. Ann Thorac Surg 2001;72:593-6.

8. Luketich JD, Schauer PR, Christie NA, Weigel TL, Raja S, et al. Minimally invasive esophagectomy. Ann Thorac Surg 2000;70:906-11.

9. Kawahara K, Maekawa T, Okabayashi K, Hideshima T, Shiraishi T, et al. Video-assisted thoracoscopic esophagectomy for esophageal 
cancer. Surg Endosc 1999;13:218-23.

10. Takeuchi H, Miyata H, Ozawa S, Udagawa H, Osugi H, et al Comparison of short-term outcomes between open and minimally invasive esophagectomy for esophageal cancer using a nationwide database in Japan. Ann Surg Oncol 2017;24:1821-7.

11. Cuschieri A, Shimi S, Banting S. Endoscopic oesophagectomy through a right thoracoscopic approach. J R Coll Surg Edinb 1992;37:7-11.

12. Akiyama H. Esophagectomy without thoracotomy. Surg Annu 1981;13:109-21.

13. Orringer MB, Sloan H. Esophagectomy without thoracotomy. J Thorac Cardiovasc Surg 1978;76:643-54.

14. Tangoku A, Yoshino S, Abe T, Hayashi H, Satou T, et al. Mediastinoscope-assisted transhiatal esophagectomy for esophageal cancer. Surg Endosc 2004;18:383-9.

15. Tokairin Y, Nagai K, Fujiwara H, Ogo T, Okuda M, et al. Mediastinoscopic subaortic and tracheobronchial lymph node dissection with a new cervico-hiatal crossover approach in thiel-embalmed cadavers. Int Surg 2015;100:580-8.

16. Tokairin Y, Nakajima Y, Kawada K, Hoshino A, Okada T, et al. A mediastinoscopic approach with bilateral cervicopneumomediastinum in radical thoracic esophagectomy. Int Surg 2017;102:278-83.

17. Tokairin Y, Nakajima Y, Kawada K, Hoshino A, Okada T, et al. The usefulness of a bilateral transcervical pneumomediastinal approach for mediastinoscopic radical esophagectomy: a right transcervical approach is an available option. Gen Thorac Cardiovasc Surg 2019;67:884-90.

18. Tokairin Y, Nakajima Y, Kawada K, Hoshino A, Okada T, et al. A feasibility study of mediastinoscopic radical esophagectomy for thoracic esophageal cancer from the viewpoint of the dissected mediastinal lymph nodes validated with thoracoscopic procedure: a prospective clinical trial. Esophagus 2019;16:214-9.

19. Fujiwara H, Shiozaki A, Konishi H, Kosuga T, Komatsu S, et al. Single-Port Mediastinoscopic Lymphadenectomy Along the Left Recurrent Laryngeal Nerve. Ann Thorac Surg 2015;100:1115-7.

20. Mori K, Aikou S, Yagi K, Nishida M, Mitsui T, et al. Technical details of video-assisted transcervical mediastinal dissection for esophageal cancer and its perioperative outcome. Ann Gastroenterol Surg 2017;1:232-7.

21. Nakajima Y, Tokairin Y, Nakajima Y, Kawada K, Nagai K, et al. Anatomical study of the left superior mediastinal lymphatics for tracheal branches of left recurrent laryngeal nerve-preserving mediastinoscope-assisted surgery in esophageal cancer. Surg Today 2018;48:333-7.

22. Murakami M, Otsuka K, Goto S, Ariyoshi T, Yamashita T, et al. Thoracoscopic and hand assisted laparoscopic esophagectomy with radical lymph node dissection for esophageal squamous cell carcinoma in the left lateral decubitus position: a single center retrospective analysis of 654 patients. BMC Cancer 2017;17:748.

23. Randolph GW, Kobler JB, Wilkins J. Recurrent laryngeal nerve identification and assessment during thyroid surgery: laryngeal palpation. World J Surg 2004;28:755-60. 